TRANSACTIONS OF THE

AMERICAN MATHEMATICAL SOCIETY

Volume 180, June 1973

\title{
ALMOST SPHERICAL CONVEX HYPERSURFACES
}

\author{
BY
}

JOHN DOUGLAS MOORE

ABSTRACT. Let $M$ be a smooth compact hypersurface with positive sectional curvatures in $n$-dimensional euclidean space. This paper gives a sufficient condition for $M$ to lie in the spherical shell bounded by concentric spheres of radius $1-\epsilon$ and $1+\epsilon$. This condition is satisfied, in the case where $n=3$, if the Gaussian curvature or the mean curvature of $M$ is sufficiently pointwise close to one.

1. Introduction. An ovaloid is a compact connected smooth surface in euclidean three-space which has positive Gaussian curvature. Well-known theorems of Liebmann [7] assert that an ovaloid whose Gaussian curvature or mean curvature is constant must be a sphere. The following question arises: If we merely require that the Gaussian curvature or mean curvature of an ovaloid be sufficiently pointwise close to a predetermined constant, does it follow that the ovaloid is close to a sphere? It clearly suffices to consider the case where the predetermined constant is one. Affirmative answers to our question are then given by the following theorems:

Theorem A. If $\epsilon>0$ is given, there exists $\delta_{1}(\epsilon)>0$ such that: If $M$ is any ovaloid whose Gaussian curvature $K$ satisfies the inequality

$$
|(1 / K)-1|<\delta_{1}(\epsilon),
$$

then $M$ must lie between two concentric spheres of radius $1-\epsilon$ and $1+\epsilon$.

Theorem B. If $\epsilon>0$ is given, there exists $\delta_{2}(\epsilon)>0$ such that: If $M$ is any ovaloid whose mean curvature $H$ satisfies the inequality

$$
|H-1|<\delta_{2}(\epsilon),
$$

then $M$ must lie between two concentric spheres of radius $1-\epsilon$ and $1+\epsilon$.

The first of the above theorems is a consequence of work of Volkov ( $[9],[8$, Chap. $7, \$ 10]$ ) on the stability of the solution to Minkowski's problem. Using the theory of elliptic partial differential equations, Koutroufiotis [6] proved analogues of Theorems A and B, under additional assumptions.

Presented to the Society, January 25, 1973; received by the editors July 28, 1972. AMS (MOS) subject classifications (1970). Primary 53C45; Secondary 53C 40.

Key words and phrases. Ovaloid, convex hypersurface, almost spherical hypersurface, integral formulae of Minkowski.

Copyright $\odot$ 1973, American Mathematical Society 
It is the purpose of this paper to describe a general approach which yields theorems similar to those mentioned above. Our main theorem, which is proven in $\$ 3$, implies Theorems $A$ and $B$ by relatively straightforward arguments. It is formulated and proven within the context of convex hypersurfaces in euclidean space of arbitrary dimension. One can therefore use the main theorem to prove various higher-dimensional generalizations of Theorems $A$ and $B$, but these will not be discussed here. Explicit proofs of Theorems $A$ and $B$ are given in $\$ 4$.

2. Preliminaries. Standard references for much of the material in this section are [3] and [4].

We let $M$ denote a smooth compact hypersurface in $n$-dimensional euclidean space $E^{n}$, where $n \geq 3$. We will assume that $M$ is strictly convex in the sense that its sectional curvatures are all positive. Then it follows from Hadamard's principle that $M$ bounds a convex body in $E^{n}$. $S^{n-1}$ will denote the sphere of unit-length vectors situated at the origin $O$ of $E^{n}$. We define a diffeomorphism $f: S^{n-1} \rightarrow M$ by stipulating that if $x \in S^{n-1}, f(x)$ must be the point on $M$ whose outward-pointing unit normal is a translate of $x$; thus $f$ is the inverse of the Gauss map. We will call $f$ the canonical parametrization of $M$. We remark that

$$
f(x) \cdot x \geq f(y) \cdot x, \text { for all } x, y \in S^{n-1},
$$

where the dot denotes the ordinary euclidean inner product.

We define the support function $b: S^{n-1} \rightarrow \mathbf{R}$ by

$$
h(x)=f(x) \cdot x, \text { for } x \in S^{n-1},
$$

so that $h(x)$ is the distance from $O$ to the tangent hyperplane to $M$ at $f(x)$. We can extend $b$ to all of $E^{n}$ by requiring that it be homogeneous of degree one. It is well known that the extended $b$ possesses the following property [3, p. 24]:

$$
b(x+y) \leq b(x)+b(y), \text { for } x, y \in E^{n} !
$$

Let $F$ denote the manifold of positively oriented orthonormal frames $\left(e_{1}, e_{2}\right.$, $\left.\cdots, e_{n}\right)$ situated at $O$ in $E^{n}, V$ the Stiefel manifold of ordered pairs $\left(e_{n-1}, e_{n}\right)$, where $e_{n-1}$ and $e_{n}$ are orthogonal unit vectors at $O$. We have the usual projections

$$
\bar{p}: F \rightarrow V, \quad p: V \rightarrow S^{n-1}
$$

defined by

$$
\bar{p}\left(e_{1}, e_{2}, \cdots, e_{n}\right)=\left(e_{n-1}, e_{n}\right), \quad p\left(e_{n-1}, e_{n}\right)=e_{n} .
$$

Observe that $V$ can be regarded as the manifold of located unit vectors tangent to $S^{n-1}$. 
We will use the following index conventions:

$$
1 \leq i, j, k \leq n-1 ; \quad 1 \leq A, B, C \leq n .
$$

We can regard each $e_{A}$ as a vector-valued function on $F$ and construct the differential forms

$$
\omega_{A}=d(f \circ p \circ \bar{p}) \cdot e_{A}, \quad \omega_{A B}=d e_{A} \cdot e_{B^{\prime}}
$$

where $\omega_{A B}=-\omega_{B A}$. Exterior differentiation of equations (2.4) yields the structure equations of Cartan:

$$
d \omega_{A}=\sum_{B} \omega_{A B} \wedge \omega_{B^{\prime}} \quad d \omega_{A B}=\sum_{C} \omega_{A C} \wedge \omega_{C B} .
$$

Since $e_{n}$ is perpendicular to $M$ at $f\left(e_{n}\right)$, we must have $\omega_{n}=0$. It follows from (2.5) that $0=d \omega_{n}=\Sigma_{i} \omega_{n i} \wedge \omega_{i}$, and hence we can use Cartan's lemma to conclude that $\omega_{i}=\Sigma_{j} b_{i j} \omega_{n j}$, where the $b_{i j}$ 's are smooth functions such that $b_{i j}=b_{j i}$. We can therefore write

$$
d(f \circ p \circ \bar{p})=\sum_{i, j} b_{i j} e_{i} \omega_{n j} .
$$

The eigenvalues of the symmetric matrix $\left(b_{i j}\right)$ are positive since $M$ is strictly convex; they are called the principal radii of curvature and will be denoted by $r_{1}, r_{2}, \cdots, r_{n-1}$. The $r_{i}$ 's will be continuous functions on $F$ if we impose the requirement that $r_{1} \leq r_{2} \leq \cdots \leq r_{n-1}$. Since $r_{i}$ is constant on the fibers of $p \circ \bar{p}$, it is the pullback via $p \circ \bar{p}$ of a continuous function on $S^{n-1}$, which will also be denoted by $r_{i}$.

We define the spherical area form $\Phi$ on $S^{n-1}$ by stipulating that

$$
\bar{p}^{*} \circ p^{*}(\Phi)=\omega_{n 1} \wedge \omega_{n 2} \wedge \cdots \wedge \omega_{n, n-1} .
$$

Similarly, we define an $(n-2)$-form $\dot{\Psi}$ on the Stiefel manifold $V$ by

$$
\bar{p}^{*}(\Psi)=\omega_{n-1,1} \wedge \cdots \wedge \omega_{n-1, n-2} .
$$

$\Psi \wedge p^{*}(\Phi)$ will be called the volume form on $V$. We orient $S^{n-1}$ and $V$ so that $\Phi$ and $\Psi \wedge p^{*}(\Phi)$ are positive. If $A$ is a Borel subset of $S^{n-1}$, we let $\Phi(A)^{\prime}$ denote its spherical area.

Let us consider the one-parameter group of diffeomorphisms $\phi_{t}$ on $V$ defined by

$$
\phi_{t}\left(e_{n-1}, e_{n}\right)=\left((\cos t) e_{n-1}-(\sin t) e_{n},(\sin t) e_{n-1}+(\cos t) e_{n}\right) .
$$

This one-parameter group is the geodesic flow for the unit sphere $S^{n-1}$ with its usual riemannian metric of constant curvature one. Indeed, if $v=\left(e_{n-1}, e_{n}\right)$ is an element of $V$, the curve $\sigma_{v}: \mathbf{R} \rightarrow S^{n-1}$ defined by $\sigma_{v}(t)=p \circ \phi_{t}(v)$ is a 
unit-speed great circle which satisfies the initial conditions $\sigma_{v}(0)=e_{n^{\prime}} \sigma_{v}^{\prime}(0)=$ $e_{n-1}$.

It is a well-known fact that the geodesic flow on a compact riemannian manifold is volume-preserving (compare $[1$, p. 3]); in other words,

$$
\phi_{t}^{*}\left(\Psi \wedge p^{*} \Phi\right)=\Psi \wedge p^{*} \Phi, \quad \text { for } t \in \mathbf{R} .
$$

This important fact can be proven in our context by a straightforward but tedious calculation.

3. The main theorem. Let $M$ and $\widetilde{M}$ be smooth compact strictly convex hypersurfaces in $E^{n}$ with canonical parametrizations $f$ and $\tilde{f}$ respectively. Using the vector-valued differential forms $d f$ and $\tilde{d f}$, we define a function $\|d f-\tilde{d f}\|$ : $S^{n-1} \rightarrow \mathbf{R}$ by setting

$$
\|d f-\tilde{d f}\|(x)=\sup \left\{|d f(v)-\tilde{d f}(u)|: v \in p^{-1}(x)\right\},
$$

where $p^{-1}(x)$ denotes the $(n-2)$-sphere of unit-length vectors tangent to $S^{n-1}$ at $x$. In the special case where $\tilde{M}$ is the unit sphere $S^{n-1}$, we can consider $\tilde{d f}$ to be the identity map id, and use (2.6) to check that

$$
\|d f-\mathrm{id}\|(x)=\sup \left\{\left|r_{i}(x)-1\right|: 1 \leq i \leq n-1\right\},
$$

where the $r_{i}(x)$ 's are the principal radii of curvature.

It is not difficult to show that if $\|d f-d \tilde{f}\|$ is uniformly small, then there exists a translation $T: E^{n} \rightarrow E^{n}$ such that $T \circ f$ is uniformly close to $\tilde{f}$. We wish to investigate what happens when we merely assume that the integral $\int_{S^{n-1}}\|d f-d \tilde{f}\| \Phi$ is small.

Main theorem. For eacb integer $n$ such that $n \geq 3$, there exists a universal constant $a_{n}$ with the following property: Let $M$ and $\bar{M}$ be smooth compact bypersurfaces in $E^{n}$ with positive sectional curvatures. Let $f$ and $\tilde{f}$ denote their canonical parametrizations, $b$ and $\tilde{b}$ their support functions. Suppose, moreover, that

$$
|\tilde{f}(x)| \leq 1, \text { for all } x \in S^{n-1} \text {. }
$$

If

$$
\int_{S^{n-1}}\|d f-\tilde{d f}\| \Phi<a_{n} \epsilon^{n}, \quad \text { where } 0<\epsilon<1
$$

then there exists a point $x_{0}$ in $E^{n}$ such that

$$
\left|b(x)+x_{0} \cdot x-\tilde{b}(x)\right|<\epsilon, \text { for all } x \in S^{n-1} .
$$

The proof of the main theorem will be given later. We remark that the func- 
tion $b(x)+x_{0} \cdot x$ which appears in (3.4) is the support function of $T(M)$, where $T$ is the translation which takes the origin to $x_{0}$. Notice that in the special case where $\tilde{M}$ is the unit sphere, hypothesis (3.2) is trivially satisfied. Hence we obtain the following

Corollary. Let $M$ be a smooth compact bypersurface in $E^{n}$ with positive sectional curvatures, and let $f$ be its canonical parametrization. If

$$
\int_{S^{n-1}}\|d f-\mathrm{id}\| \Phi<a_{n} \epsilon^{n}, \text { where } 0<\epsilon<1
$$

then there exists a point $x_{0}$ in $E^{n}$ such that

$$
\left|f(x)-\left(x-x_{0}\right)\right|<\epsilon, \text { for all } x \in S^{n-1} .
$$

Thus $M$ lies between two spheres which are centered at $-x_{0}$ and have radii $1-\epsilon$ and $1+\epsilon$.

Proof of the Corollary. The main theorem gives us a point $x_{0}$ in $E^{n}$ such that $\left|\left(f(x)+x_{0}\right) \cdot x-1\right|<\epsilon$, for $x \in S^{n-1}$. It follows from (2.1) that $\left(f(x)+x_{0}\right) \cdot y$ $\leq\left(f(y)+x_{0}\right) \cdot y<1+\epsilon$, for $x, y \in S^{n-1}$, and hence $\left|f(x)+x_{0}\right|<1+\epsilon$. Thus we find that

$$
\left|f(x)+x_{0}-x\right|^{2}=\left|f(x)+x_{0}\right|^{2}-2\left(f(x)+x_{0}\right) \cdot x+1<(1+\epsilon)^{2}-2(1-\epsilon)+1=\epsilon^{2},
$$

which yields (3.6) and proves the corollary.

To facilitate the proof of the main theorem, we introduce some notation. Suppose that we are given canonical parametrizations $f$ and $\tilde{f}$ of smooth compact strictly convex hypersurfaces in $E^{n}$. For each translation $T$ of $E^{n}$ and each positive number $\delta$, let

$$
\begin{aligned}
& A(T, \delta)=\left\{x \in S^{n-1}:|T \circ f(x)-\tilde{f}(x)|<\delta\right\}, \\
& B(T, \delta)=\left\{x \in S^{n-1}:|T \circ f(x)-\tilde{f}(x)| \geq \delta\right\} .
\end{aligned}
$$

Under the hypotheses of the main theorem, we will seek a translation $T$ such that the measure of $B(T, \delta)$ is small. Let $A_{m}$ denote the area of the $m$-dimensional unit sphere, and set

$$
b_{n}=\left(\pi^{2} A_{n-2}\right) / A_{n-1} .
$$

Definition. We will say that a translation $T$ of $E^{n}$ is admissible for the pair $(f, \tilde{f})$ if

$$
\Phi(B(T, \delta)) \leq-\frac{b_{n}}{\delta} \int_{s^{n-1}}\|d f-\tilde{d f}\| \Phi, \quad \text { for all } \delta>0 .
$$


Lemma. If $f$ and $\tilde{f}$ are canonical parametrizations of smootb compact bypersurfaces in $E^{n}$ with positive sectional curvatures, then there exists an admissible translation $T$ for the pair $(f, \tilde{f})$.

Proof. Let

$$
\Delta=\int_{S^{n-1}}\|d f-\tilde{d f}\| \Phi
$$

then $\int_{V}(\|d f-d f\| \circ p)\left(\Psi \wedge p^{*} \Phi\right)=A_{n-2} \Delta$. Since the diffeomorphism $\phi_{t}$ is volume-preserving,

$$
\int_{V}\left[\int_{0}^{\pi}\left(\|d f-\tilde{d f}\| \circ p \circ \phi_{t}\right) d t\right]\left(\Psi \wedge p^{*} \Phi\right)=\pi A_{n-2} \Delta
$$

The integration over $V$ can be performed by first integrating over the fibers of $p$. and then integrating over the base (compare [5, pp. 57, 58]). It follows that there exists a point $z \in S^{n-1}$ such that

$$
\int_{p-1(z)}\left[\int_{0}^{\pi}\left(\|d f-\tilde{d f}\| \circ p \circ \phi_{t}\right) d t\right] \Psi \leq\left(\pi A_{n-2} \Delta\right) / A_{n-1},
$$

where $p^{-1}(z)$ is oriented so that $\Psi$ is positive. We choose such a point $z$, and leave it fixed for the remainder of the argument. Then we select our translation $T$ so that

$$
T \circ f(z)=\tilde{f}(z) .
$$

Let us consider the smooth mapping $\psi: p^{-1}(z) \times[0, \pi] \rightarrow s^{n-1}$ defined by $\psi(v, t)=p \circ \phi_{t}(v)$, which is clearly surjective. A straightforward calculation shows that

$$
\psi^{*} \Phi=\left(\sin ^{n-2} t\right)\left(q^{*} \Psi\right) \wedge d t
$$

where $q$ is the natural projection from $p^{-1}(z) \times[0, \pi]$ to $p^{-1}(z)$.

We introduce the sets

$$
\begin{aligned}
& \tilde{A}(\delta)=\left\{v \in p^{-1}(z): \int_{0}^{\pi}[\|d f-\tilde{d f}\| \circ \psi(v, t)] d t<\delta\right\}, \\
& \widetilde{B}(\delta)=\left\{v \in p^{-1}(z): \int_{0}^{\pi}[\|d f-\tilde{d f}\| \circ \psi(v, t)] d t \geq \delta\right\} .
\end{aligned}
$$

If $x=\psi(v, t)$, where $(v, t) \in \tilde{A}(\delta) \times[0, \pi]$, then we can use (3.13) to show that

$$
\begin{aligned}
|T \circ f(x)-\tilde{f}(x)| & \leq|T \circ f(z)-\tilde{f}(z)|+\int_{0}^{t}\left[\|d f-\tilde{d f}\| \circ p \circ \phi_{s}(v)\right] d s \\
& \leq \int_{0}^{\pi}[\|d f-\tilde{d f}\| \circ \psi(v, t)] d t<\delta .
\end{aligned}
$$

It follows that $\psi(\tilde{A}(\delta) \times[0, \pi]) \subseteq A(T, \delta)$, from which we can draw the following conclusion: 


$$
B(T, \delta) \subseteq \psi(\tilde{B}(\delta) \times[0, \pi])
$$

By definition of $\widetilde{B}(\delta)$, we must have $\int \widetilde{B}(\delta)\left[\int_{0}^{\pi}\left(\|d f-d \tilde{f}\| \circ p \circ \phi_{t}\right) d t\right] \Psi \geq$ $\delta \int_{B}(\delta) \Psi$. Hence it follows from inequality (3.12) that

$$
\int_{\widetilde{B}(\delta)} \Psi \leq\left(\pi A_{n-2} \Delta\right) /\left(A_{n-1} \delta\right) \text {. }
$$

We now apply (3.14), (3.15), and (3.16) in succession:

$$
\begin{aligned}
\Phi(B(T, \delta)) & =\int_{\psi^{-1}(B(T, \delta))}\left(\sin ^{n-2} t\right)\left(q^{*} \Psi\right) \wedge d t \\
& \leq \int_{\widetilde{B}(\delta) \times[0, \pi]}\left(q^{*} \Psi\right) \wedge d t \leq\left(\pi^{2} A_{n-2} \Delta\right) /\left(A_{n-1} \delta\right)=b_{n} \Delta / \delta
\end{aligned}
$$

In view of (3.11), this proves the lemma.

Proof of the main theorem. First of all we will specify the constant $a_{n}$. If $x \in S^{n-1}$ and $\delta \in[0, \pi / 2]$, we let $D(x, \delta)=\left\{y \in S^{n-1}: x \cdot y>\cos \delta\right\}$; thus $D(x, \delta)$ consists of the points in $S^{n-1}$ whose great circle distance from $x$ is less than $\delta$. An elementary calculation gives an estimate for the measure of $D(x, \delta)$ :

$$
\Phi(D(x, \delta))=A_{n-2} \int_{0}^{\delta} \sin ^{n-2} t d t
$$

$$
\geq A_{n-2} \int_{0}^{\delta}(2 t / \pi)^{n-2} d t=c_{n} \delta^{n-1}, \text { for } 0 \leq \delta \leq \pi / 2
$$

where $c_{n}$ is a constant. We set

$$
a_{n}=c_{n} /\left(2 \cdot 4^{n} b_{n}\right) .
$$

Suppose now that $f$ and $\tilde{f}$ are canonical parametrizations of smooth compact strictly convex hypersurfaces in $E^{n}$ which satisfy (3.2) and (3.3). By the previous lemma, there exists an admissible translation $T$ for the pair $(f, \tilde{f})$. We select such a translation $T$ and set $x_{0}=T(0)$.

Let $x$ be an arbitrary point of $S^{n-1}$, which we will leave fixed until the end of the proof. If we set $\delta=\epsilon / 4$, it follows from (3.10), (3.3), and (3.18) that

$$
\Phi(B(T, \delta)) \leq\left(b_{n} / \delta\right) \int_{S^{n-1}}\|d f-\tilde{d f}\| \Phi<\left(a_{n} b_{n} \epsilon^{n}\right) / \delta=\left(c_{n} \delta^{n-1}\right) / 2
$$

Since $\delta<\pi / 2$, (3.17) yields the inequality $\Phi(B(T, \delta))<1 / 2 \Phi(D(x, \delta))$, which in turn allows us to conclude that

$$
\Phi(A(T, \delta) \cap D(x, \delta))>1 / 2 \Phi(D(x, \delta)) .
$$

Inequality (3.19) implies that there exist points $y, z$ in the intersection $A(T, \delta) \cap D(x, \delta)$ such that $x=(y+z) /|y+z|$. Since $y$ and $z$ are members of $A(T, \delta)$ it follows from (3.7) that

$$
\left|f(y)+x_{0}-\tilde{f}(y)\right|<\delta, \quad\left|f(z)+x_{0}-\tilde{f}(z)\right|<\delta .
$$


On the other hand, the fact that $y$ and $z$ lie in $D(x, \delta)$ implies that

$$
|y-x|<\delta, \quad|z-x|<\delta .
$$

Since $y \cdot z>\cos (2 \delta) \geq 1-2 \delta^{2}$, we can find an upper bound for $1 /|y+z|$. Indeed, $|y+z|^{2}=2(1+y \cdot z)>4\left(1-\delta^{2}\right)$, and our assumption that $0<\epsilon<1$ allows us to derive the estimate

$$
|y+z|^{-1}<1 / 2\left(1-(\epsilon / 4)^{2}\right)^{-1 / 2}<1 / 2(1+(\epsilon / 4))=1 / 2(1+\delta) .
$$

We now put the various inequalities together:

$$
\begin{aligned}
& b(x)+x_{0} \cdot x \leq|y+z|^{-1}\left[b(y)+b(z)+x_{0} \cdot y+x_{0} \cdot z\right] \\
& \text { (2.3) } \\
& \underset{(3.22)}{<} 1 / 2(1+\delta)\left[b(y)+b(z)+x_{0} \cdot y+x_{0} \cdot z\right] \\
& \underset{(3.20)}{<} 1 / 2(1+\delta)[\tilde{b}(y)+\tilde{b}(z)+2 \delta] \\
& =1 / 2(1+\delta)[\tilde{f}(y) \cdot x+\tilde{f}(y) \cdot(y-x)+\tilde{f}(z) \cdot x+\tilde{f}(z) \cdot(z-x)+2 \delta] \\
& \underset{(3.2) \&(3.21)}{\leq} 1 / 2(1+\delta)[\tilde{f}(y) \cdot x+\tilde{f}(z) \cdot x+4 \delta] \underset{(2.1)}{\leq} 1 / 2(1+\delta)[2 \tilde{f}(x) \cdot x+4 \delta] \\
& \leq \widetilde{b}(x)+3 \delta+2 \delta^{2}=\tilde{b}(x)+3 \epsilon / 4+\epsilon^{2} / 8<\tilde{b}(x)+\epsilon . \\
& \text { (3.2) }
\end{aligned}
$$

On the other hand,

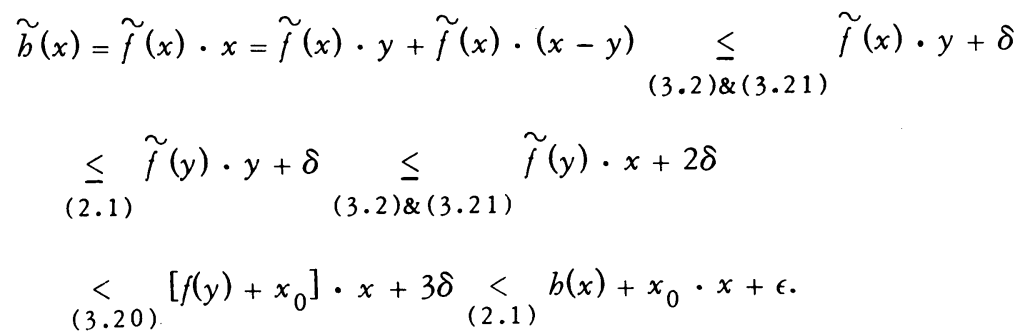

This establishes (3.4) and finishes the proof of the main theorem.

4. Applications: almost spherical ovaloids. In this section we will give some applications of the corollary of the main theorem; namely, we will prove Theorems $A$ and $B$ of the Introduction. Throughout this section we will assume that $n=3$, and $M$ will denote a smooth ovaloid in ordinary euclidean three-space.

We let $2 \sigma_{1}$ and $\sigma_{2}$ be the elementary symmetric functions of the principal 
radii of curvature of $M$; then we have the equations

$$
\sigma_{1}=1 / 2\left(r_{1}+r_{2}\right)=H / K, \quad \sigma_{2}=r_{1} r_{2}=1 / K,
$$

where $K$ is the Gaussian curvature of $M$, and $H$ is the mean curvature with respect to the inward-pointing unit normal. We recall the inequality

$$
\sigma_{1}^{2} \geq \sigma_{2}
$$

Since $r_{i}=\sigma_{1} \pm \sqrt{\sigma_{1}^{2}-\sigma_{2}}$, it follows from (3.1) that

$$
\|d f-\mathrm{id}\|=\left|\sigma_{1}-1\right|+\sqrt{\sigma_{1}^{2}-\sigma_{2}} .
$$

Our proofs of Theorems A and B will be based upon the following fundamental integral formulae of Minkowski, which are proven in [4, p. 114]:

$$
\int_{S^{2}} \sigma_{1} \Phi=\int_{S^{2}} b \Phi, \quad \int_{S^{2}} \sigma_{2} \Phi=\int_{S^{2}} b \sigma_{1} \Phi
$$

Proof of Theorem A. Suppose that $M$ is an ovaloid whose Gaussian curvature $K$ satisfies inequality (1.1), where $0<\delta_{1}(\epsilon)<1$. We set $\delta=\delta_{1}(\epsilon)$, and rewrite this inequality as

$$
1-\delta<\sigma_{2}<1+\delta, \text { where } 0<\delta<1 .
$$

It follows from (4.2) that

$$
\sigma_{1}>\sqrt{1-\delta}
$$

We can assume without loss of generality that the origin lies inside $M$, so that $b>0$. Then

$$
\begin{aligned}
\int_{S^{2}} \sigma_{1} \Phi \underset{(4.4)}{=} \int_{s^{2}} b \Phi \underset{(4.6)}{<} \frac{1}{\sqrt{1-\delta}} \int_{s^{2}} b \sigma_{1} \Phi \\
\underset{(4.4)}{=} \frac{1}{\sqrt{1-\delta}} \int_{S^{2}} \sigma_{2} \Phi \underset{(4.5)}{<} \frac{1+\delta}{\sqrt{1-\delta}} \int_{s^{2}} \Phi
\end{aligned}
$$

which yields the inequality

$$
\int_{S^{2}}\left(\sigma_{1}-\sqrt{1-\delta}\right) \Phi<\frac{2 \delta}{\sqrt{1-\delta}} \int_{S^{2}} \Phi=\frac{8 \pi \delta}{\sqrt{1-\delta}}=g_{1}(\delta)
$$

where $g_{1}(\delta)$ is a function which approaches zero as $\delta$ goes to zero. Since $\sigma_{1}-\sqrt{1-\delta}$ is positive by $(4.6)$, it follows from $(4.7)$ that

$$
\int_{S^{2}}\left|\sigma_{1}-1\right| \Phi<g_{1}(\delta)+4 \pi(1-\sqrt{1-\delta})=g_{2}(\delta),
$$

where $g_{2}(\delta) \rightarrow 0$ as $\delta \rightarrow 0$. By (4.5), $\sigma_{1}^{2}-\sigma_{2}<\sigma_{1}^{2}-(1-\delta)=\left[\sigma_{1}-\sqrt{1-\delta}\right]$. $\left[\sigma_{1}+\sqrt{1-\delta]}\right.$. We use the Cauchy-Schwarz inequality and (4.7) to conclude that 


$$
\begin{aligned}
\int_{S^{2}} \sqrt{\sigma_{1}^{2}-\sigma_{2}} \Phi & <\left[\int_{S^{2}}\left(\sigma_{1}-\sqrt{1-\delta}\right) \Phi\right]^{1 / 2}\left[\int_{S^{2}}\left(\sigma_{1}+\sqrt{1-\delta}\right) \Phi\right]^{1 / 2} \\
& <\left[g_{1}(\delta)\left(g_{1}(\delta)+8 \pi \sqrt{1-\delta}\right)\right]^{1 / 2}=g_{3}(\delta) .
\end{aligned}
$$

Now (4.3), (4.8), and (4.9) yield the inequality

$$
\int_{S^{2}}\|d f-\mathrm{id}\| \Phi<g_{2}(\delta)+g_{3}(\delta)=g(\delta),
$$

where $g(\delta) \rightarrow 0$ as $\delta \rightarrow 0$.

If $\epsilon>0$ is given, choose $\delta$ so that $0<\delta<1$ and $g(\delta)<a_{3} \epsilon^{3}$. If $M$ is an ovaloid whose Gaussian curvature satisfies (4.5), it follows from (4.10) and the corollary of the main theorem that $M$ lies between two concentric spheres with radii $1-\epsilon$ and $1+\epsilon$. This proves Theorem $A$.

Proof of Theorem B. Suppose that $M$ is an ovaloid whose mean curvature $H$ satisfies inequality (1.2), where $0<\delta_{2}(\epsilon)<1$. We set $\delta=\delta_{2}(\epsilon)$, and rewrite the inequality as

$$
1-\delta<\sigma_{1} / \sigma_{7}<1+\delta, \text { where } 0<\delta<1 .
$$

Using (4.2) we find that

$$
\sigma_{1}<(1+\delta) \sigma_{2} \leq(1+\delta) \sigma_{.1}^{2} \Rightarrow \sigma_{1}>1 /(1+\delta),
$$

and hence

$$
\sigma_{2}>\sigma_{1} /(1+\delta)>1 /(1+\delta)^{2}
$$

Since $r_{1}$ and $r_{2}$ are positive, and $\left(1 / r_{1}\right)+\left(1 / r_{2}\right)=2 H<2(1+\delta)$, we must have

$$
r_{i}>1 /(2+2 \delta)>1 / 4 \text {. }
$$

A theorem of Blaschke [2, p. 118] as serts that if the principal radii of curvature of an ovaloid are bounded below by a positive constant $c$, then the ovaloid contains a sphere of radius $c$. Thus in our case it follows from inequality (4.14) that $M$ contains a sphere of radius $1 / 4$. We can assume without loss of generality that the origin is at the center of this sphere, so that

$$
b \geq 1 / 4 \text {. }
$$

Now we seek an estimate for the integral of the positive function $\sigma_{1}-1 /(1+\delta)$ :

$$
\begin{aligned}
& \int_{S^{2}}\left[\sigma_{1}-1 /(1+\delta)\right] \Phi \underset{(4.15)}{\leq} 4 \int_{S^{2}}\left[h \sigma_{1}-b /(1+\delta)\right] \Phi \\
& \underset{(4.4)}{=} 4 \int_{S^{2}}\left[\sigma_{2}-\sigma_{1} /(1+\delta)\right] \Phi \underset{(4.11)}{<} 4 \int_{S^{2}}\left[\sigma_{1} /(1-\delta)-\sigma_{1} /(1+\delta)\right] \Phi \\
& =\left[8 \delta /\left(1-\delta^{2}\right)\right] \int_{S^{2}} \sigma_{1} \Phi=\left[8 \delta /\left(1-\delta^{2}\right)\right]\left\{\int_{S^{2}}\left[\sigma_{1}-1 /(1+\delta)\right] \Phi+4 \pi /(1+\delta)\right\} .
\end{aligned}
$$


This yields the inequality

$$
\left[1-8 \delta /\left(1-\delta^{2}\right)\right] \int_{S^{2}}\left[\sigma_{1}-1 /(1+\delta)\right] \Phi<32 \pi \delta /(1-\delta)(1+\delta)^{2},
$$

which can be rewritten in the form

$$
\int_{S^{2}}\left[\sigma_{1}-1 /(1+\delta)\right] \Phi<g_{1}(\delta),
$$

where $g_{1}(\delta) \rightarrow 0$ as $\delta \rightarrow 0$.

Since the integrand in (4.16) is positive, it follows that

$$
\int_{S^{2}}\left|\sigma_{1}-1\right| \Phi<g_{1}(\delta)+4 \pi[1-1 /(1+\delta)]=g_{2}(\delta) .
$$

From (4.13), we obtain the inequality

$$
\sigma_{1}^{2}-\sigma_{2}<\sigma_{1}^{2}-1 /(1+\delta)^{2}=\left[\sigma_{1}-1 /(1+\delta)\right]\left[\sigma_{1}+1 /(1+\delta)\right] .
$$

We use this together with the Cauchy-Schwarz inequality and (4.16) to conclude that

$$
\int_{S^{2}} \sqrt{\sigma_{1}^{2}-\sigma_{2}} \Phi<\left[\int_{S^{2}}\left[\sigma_{1}-1 /(1+\delta)\right] \Phi\right]^{1 / 2} \int_{S^{2}}\left[\left[\sigma_{1}+1 /(1+\delta)\right] \Phi\right]^{1 / 2}
$$

$$
<\left[g_{1}(\delta)\left(g_{1}(\delta)+8 \pi /(1+\delta)\right)\right]^{1 / 2}=g_{3}(\delta) .
$$

Now (4.3), (4.17), and (4.18) yield the inequality

$$
\int_{S^{2}}\|d f-\mathrm{id}\| \Phi<g_{2}(\delta)+g_{3}(\delta)=g(\delta)
$$

where $g(\delta) \rightarrow 0$ as $\delta \rightarrow 0$.

Given $\epsilon>0$, choose $\delta$ so that $0<\delta<1$ and $g(\delta)<a_{3} \epsilon^{3}$. If $M$ is an ovaloid whose mean curvature satisfies the inequality $|H-1|<\delta$, then it follows from (4.19) and the corollary of the main theorem that $M$ lies in the spherical shell bounded by concentric spheres with radii $1-\epsilon$ and $1+\epsilon$. This proves Theorem B.

\section{REFERENCES}

1. V. I. Arnol'd and A. Avez, Ergodic problems of classical mechanics, GauthierVillars, Paris, 1967; English transl.,, Benjamin, New York, 1968. MR 35 \#334; MR 38 $\# 1233$.

2. W. Blaschke, Kreis and Kugel, Viet, Leipzig, 1916; reprint, Chelsea, New York, 1949. MR 17, 887.

3. T. Bonnesen and W. Fenchel, Theorie der Konvexer Körper, Springer, Berlin, 1934; reprint, Chelsea, New York, 1948.

4. H. Flanders, Differential forms with applications to the physical sciences, Academic Press, New York, 1963. MR 28 \# 5397.

5. R. Hermann, Differential geometry and the calculus of variations, Math. in Sci. 
and Engineering, vol. 49, Academic Press, New York, 1968. MR 38 \# 1635.

6. D. Koutroufiotis, Ovaloids which are almost spheres, Comm. Pure Appl. Math. 24 (1971), 289-300. MR $43 \# 8030$.

7. H. Liebmann, Über die Verbiegung der geschlossenen Flächen positiver Krümmung, Math. Ann. 53 (1900), $81-112$.

8. A. V. Pogorelov, The extrinsic geometry of convex surfaces, "Nauka", Moscow, 1969; English transl., Transl. Math. Monographs, vol. 35, Amer. Math. Soc., Providence, R.I., 1973. MR 39 \#6222.

9. Ju. A. Volkov, Stability of the solution of Minkowski's problem, Vestnik Leningrad. Univ. Ser. Mat. Meh. Astronom. 18 (1963), no. 1, 33-43. (Russian) MR 26 \# 4258.

DEPARTMENT OF MATHEMATICS, UNIVERSITY OF CALIFORNIA, SANTA BARBARA, CALIFORNIA 93106 